\title{
Optical and structural properties of PVA films doped with $\mathrm{Cu}$ nanoparticles
}

\author{
Rezhna Mhammed K.M. Mahmood M. Kareem. Dana M. Mhammed \\ Physics Department, College of education, Garmian University, Kurdistan Region, Iraq.
}

\begin{abstract}
Optical and structural properties of composite films are studied with the effect of $\mathrm{Cu}$ nanoparticles doped in PVA. Samples are PVA films of $(0.13 \mathrm{~mm})$ thickness are doped with different concentrations of $\mathrm{Cu}$ nanoparticles. The nano-composite samples are prepared by the well-known solution cast method. $\mathrm{Cu}$ nanoparticles are prepared by laser ablation method using Nd-Yag laser in pure water. Using the absorbance and transmittance of the samples, the effect of doped nanoparticles and their concentration on optical parameters of PVA films including absorption coefficient, optical band gap energy, complex refractive index, complex dielectric function, before and after annealing are extracted and discussed. Studying the effect of $\mathrm{Cu}$ nanoparticles on the optical parameters gives an indication that all these parameters are affected. Also the optical properties of the films are highly affected by annealing. $\mathrm{Cu}-\mathrm{O}$ bonds are formed in the films and the band gap energy of samples is decreased. Structural properties are studied using X-ray diffraction spectrum. The results show that by doping $\mathrm{Cu}$ nanoparticles in PVA, The intensity of diffracted X-ray photons from films has been increased noticeably after the doping process.
\end{abstract}

Keywords: polyvinyl alcohol (PVA) films, X-ray diffraction, Bragg's plane, $\mathrm{Nd-}$ Yag laser, Cu nanoparticles, Doping, Optical properties, nano-composite.

\section{Introduction}

It is well established that polymers, as dielectric materials, are excellent host matrices for encapsulation of metal nanoparticles like silver, gold, copper, and so forth, as they act both as reducing as well as capping agents and also provide environmental and chemical stability [1-2]. Many reports in the literature show attempts for synthesis of metal nanoparticles based polymer nano-composites, with the possibility of variation in their optical and electrical properties for their 
application in high performance capacitors, conductive inks, and other electronic components [3, 4, and 5]. Special worthy has been reached to optical properties of the nanoparticles doped in polymer film, depending on the surrounding medium [6-7] and on their size, shape, and concentration [8-9].

Since copper is the most widely used material due to its low cost and very high electrical and thermal conductivities, $\mathrm{Cu}$ nano- particles have attracted a great deal of attention of researchers. Therefore, during recent years, the synthesis of $\mathrm{Cu}$ nanoparticles has become a subject of great interest from scientific as well as industrial point of view. Poly (vinyl alcohol) (PVA) is a polymer with high transparency in the visible spectral domain and has wide range of potential applications in optical, pharmaceutical, medical, and membrane fields. In fact it is a water soluble polymer and allows the development of environment-friendly material processes [11-12]. PVA can effectively protect the nanoparticles from aggregation $[10,2]$. This polymer is a good candidate for incorporation into multilayer coatings of organic solar cells due to its high transparency and ability to form a barrier to oxygen [13]. In this research paper, we focus on the structural and optical properties variation of the supporting polymer due to $\mathrm{Cu}$ nanoparticles doping and annealing effect. $\mathrm{Cu}$ nanoparticles were produced by laser ablation method. The laser ablation technique in a liquid produces proper metal nanoparticle samples to facilitate investigation of their photo physical and photochemical properties [14].

Formation of $\mathrm{Cu}$ nanoparticles was confirmed using UV-Visible Spectroscopy, Xray Diffraction technique (XRD). The response transmittance, absorbance, reflection, optical band gap, dielectric constant, optical conductivity, refractive index, of PVA-Cu nanoparticles films at room temperature with varying concentration of $\mathrm{Cu}$ NPs at the same thickness of $\mathrm{Cu}$ nanoparticles doped PVA films are also investigated before and after annealing.

\section{Materials and Method}


The PVA powder was provided by (BDH chemicals, England) with high purity (99.999\%). PVA films were prepared by dissolving $1 \mathrm{~g}$ of PVA powder in $20 \mathrm{~mL}$ pure water at $75 \mathrm{C}^{\circ}$. The mixture was stirred for one hour continuously to form a viscous solution. Nanoparticles (NPs) were prepared by ablation of a high purity $\mathrm{Cu}$ bulk in pure water, using Nd: YAG laser system operating at $1064 \mathrm{~nm}$ with 2 $\mathrm{Hz}$ repetition rate. $\mathrm{Cu}$ bulk was placed at the bottom of a water container height of water on the $\mathrm{Cu}$ target was $14 \mathrm{~mm}$. The volume of the water in the ablation container was $5 \mathrm{~mL}$ and $\mathrm{Cu}$ target was ablated with 800 laser pulses at different energies $(800 \mathrm{~mJ}, 1200 \mathrm{~mJ}, 1800 \mathrm{~mJ})$. Samples $1-3$ were prepared with laser pulse respectively. After completing desolation of PVA, $5 \mathrm{~mL}$ of $\mathrm{Cu}$ nanoparticles suspension was added to the $20 \mathrm{~mL}$ aqueous PVA solution, and finally, samples was left to dry on a plane surface for $24 \mathrm{hr}$. at room temperature in close atmosphere to produce 4 samples of $0.13 \mathrm{~mm}$ thickness Sample 1 is pure PVA and 3 uniform $\mathrm{Cu}$ NPs doped PVA films. S2 to S4 are PVA films which are doped with samples 1 to 3 nanoparticles. The X-ray diffraction (XRD) patterns of un-doped and doped PVA films were measured employing STOE-XRD diffract meter with $\mathrm{Cu}-\mathrm{K} \alpha$ radiation $(\lambda=1.54 \mathrm{~A})$. the samples where annealed for one hour in $75{ }^{\circ} \mathrm{C}$ .The absorbance and transmittance spectra were recorded for samples before and after annealing using double beam Schimadzu UV/VIS- 160A in the wavelength range (190-1100) $\mathrm{nm}$; the measurements were carried out at room temperature. A comparison is done between the sample before and after annealing.

\section{Result and Calculation}

Nanoparticle samples are shown in Figures 1 which are prepared by laser ablation method. The variation of transmittance $(T)$ and absorbance (A) as a function of 
wavelength of the incident light before annealing for the pure PVA and PVA doped samples were recorded at room temperature and are illustrated in Fig. 2(a)-2(b).

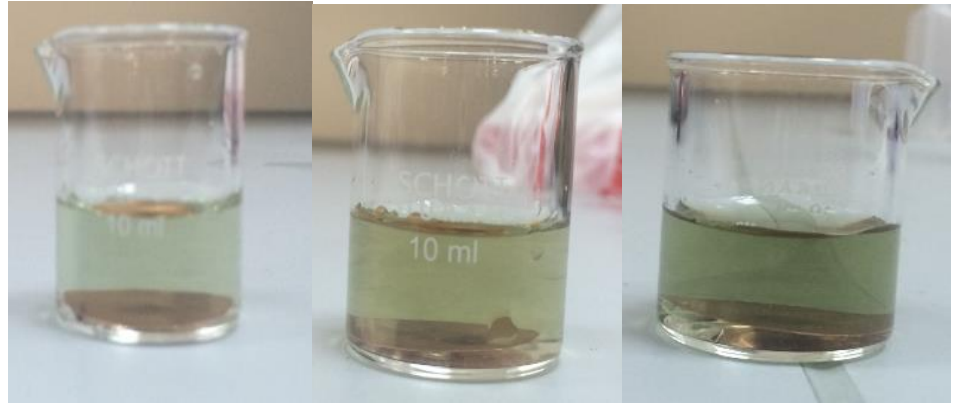

Figure 1: $\mathrm{Cu}$ nanoparticle samples in distilled water

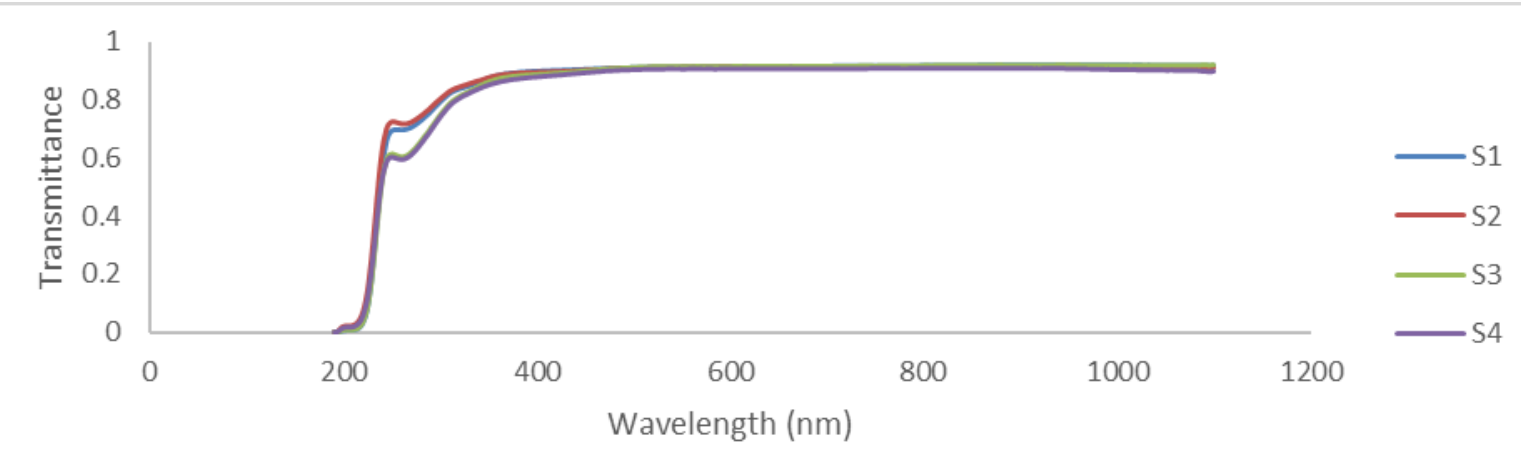

(a)

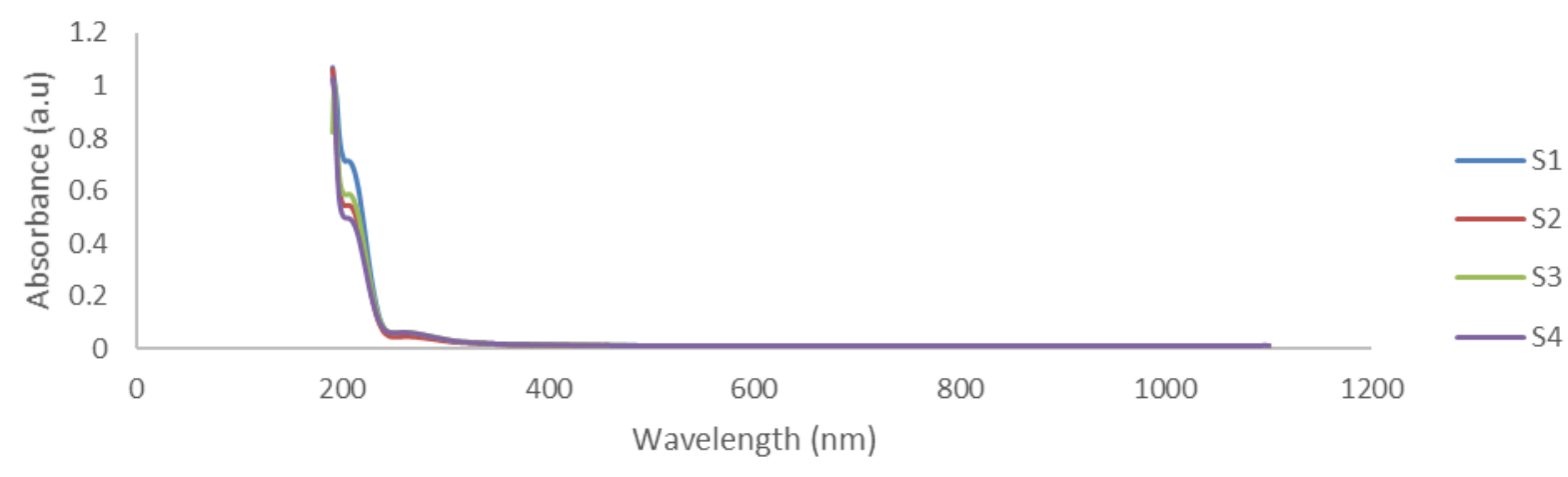

(b)

Fig. 2. UV-Visible spectra before annealing for pure PVA and Cu-PVA nanocomposite films

(a) Transmittance (T), (b) Absorbance (A)

It is clear that before annealing the transmittance has a maximum value in the wavelength range of (400-800) $\mathrm{nm}$ for PVA and transparency falls to nearly $80 \%$ in the $\mathrm{Cu}$-PVA nanocomposite film. This is due to the formation of intermolecular hydrogen bonding between $\mathrm{Cu}$ ions and the adjacent $\mathrm{OH}$ groups [15]. From the figures it can be noticed that before annealing the absorbance for all films have a high values at wavelength in the neighborhood of the fundamental absorption 
edge $(200 \mathrm{~nm})$, then the absorbance decreases with the increasing of wavelength. These results are in agreement with the researchers $[16,17]$.

The absorption coefficient $\alpha$ was calculated from the absorbance A by using the following equation [18].

$\alpha=\frac{2.303 A}{x}$

Where: $\mathrm{x}$ is the film thickness, $\mathrm{A}$ is the absorbance.

Fig.(3): shows the variation of absorption coefficient $(\alpha)$ with photon energy before annealing for the pure PVA and doped PVA nanocomposite films. It is clear that the absorption edge of PVA shifts to lower photon energy upon the addition of $\mathrm{Cu}$ nanoparticles, due to the decrease in the optical band gap for the doped samples.

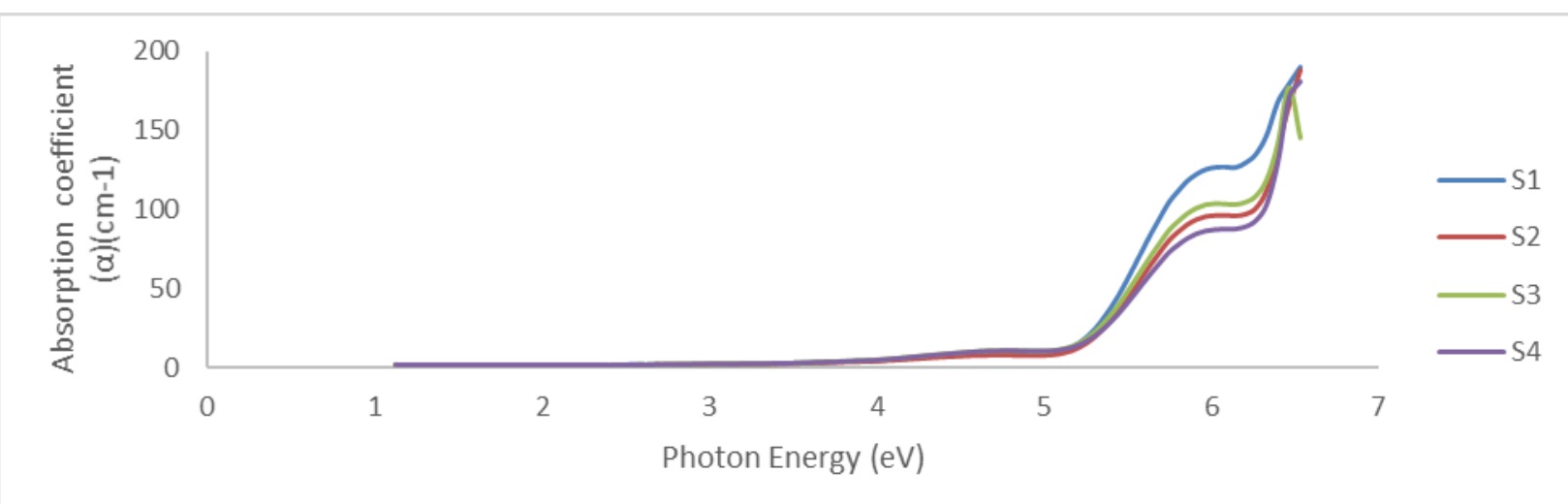

Figure (3): The absorption coefficient $\alpha(\mathrm{cm})-1$ as a function photon energy $(\mathrm{h} v)$ before annealing for pure PVA and PVA doped nanocomposite films

Band gap can be determined from the absorption coefficient according to [19]:

$\alpha(v) h v=B\left(h v-E_{g}\right)^{m}$

Where $\mathrm{h} v$ is the photon energy, $\alpha$ is the absorption coefficient, $\mathrm{E}_{\mathrm{g}}$ is the optical band gap, $B$ is band tailing parameter and $m=1 / 2$ for direct band gap and $m=2$ for indirect band gap. When the value of $\mathrm{m}=2$, the allowed indirect transition band energy gap is calculated, but when the value of $\mathrm{m}=3$, the forbidden indirect transition band energy gap is calculated. Figure (3) show the relation between absorption edge $(\alpha h v)^{1 / 2}$ for pure PVA and PVA doped nanocomposite films as a function of photon energy before annealing, on drawing straight line from the 
upper part of the curve toward the $(\mathrm{x})$ axis at the value $(\alpha \mathrm{hv})^{1 / 2}=0$ we get the energy gap for the allowed indirect transition.

It can be noticed from Fig. 4 that after extrapolation of the fitting lines, the value of optical band gap before annealing comes out to be $6.20 \mathrm{eV}$ for pure PVA and it decreases to a value of $6.00 \mathrm{eV}$ for PVA doped nanocomposite films (S2). With further increase in concentration of $\mathrm{Cu}(\mathrm{S} 3)$, it decreases to a value of $5.90 \mathrm{eV}$. When the concentration of $\mathrm{Cu}$ is further increased (S4), the value of band gap further decreases to $5.80 \mathrm{eV}$ for the PVA doped nanocomposite films. We can see that the values of energy gap decrease with increasing concentration of Cu NPs.

The variation of the calculated values of optical band gap reflects the role of formation of $\mathrm{Cu}$ nanoparticles in modifying the electronic structure of the PVA matrix [22]. These $\mathrm{Cu}$ nanoparticles may be responsible for the formation of localized electronic states in the Highest Occupied Molecular Orbital-Lowest Unoccupied Molecular Orbital (HOMO-LUMO) gap.

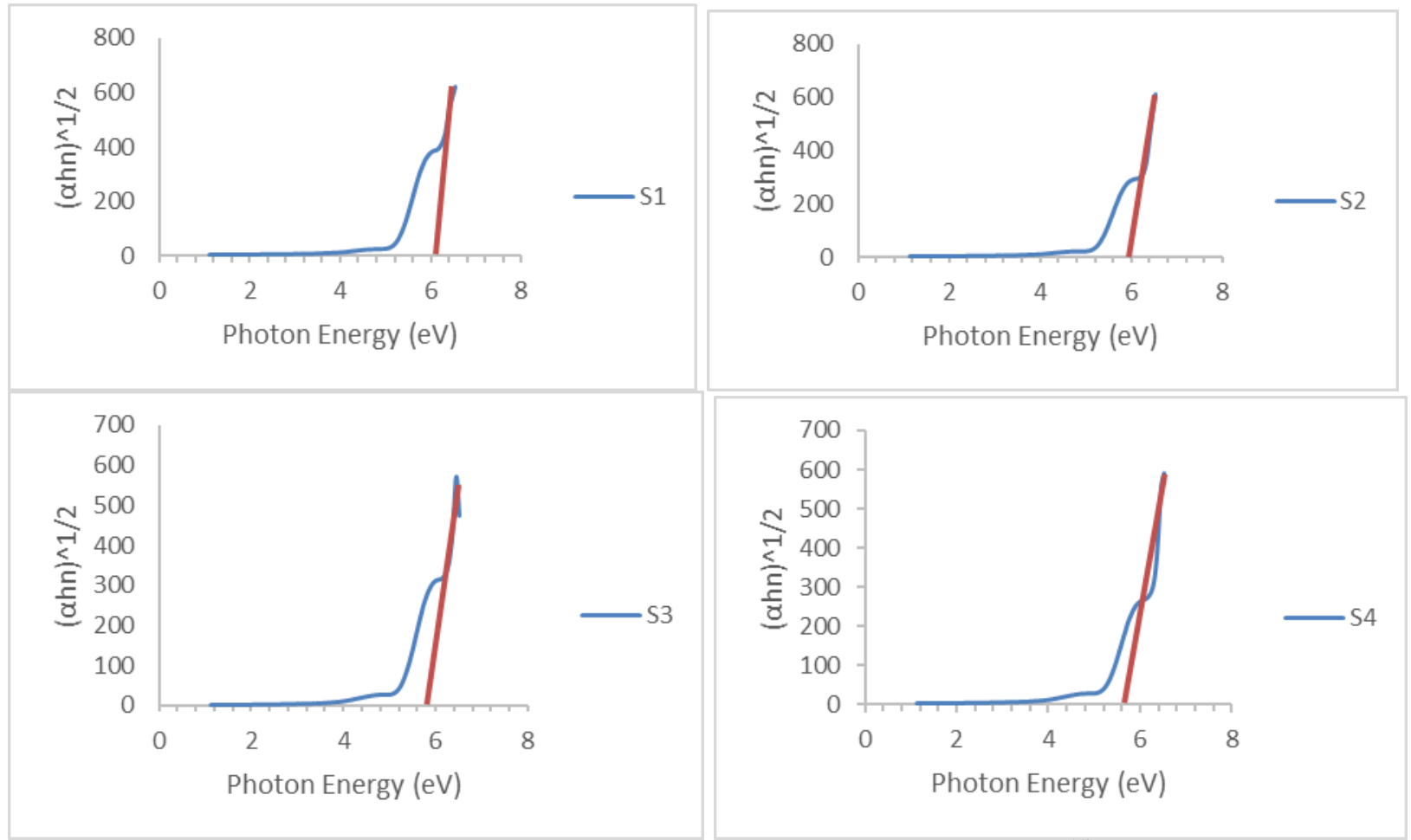

Figure (4): The energy gap before annealing for the allowed indirect transition $(\alpha h v)^{1 / 2}$ as a function of photon energy of pure PVA and PVA doped nanocomposite films. 
To further understand the interaction of $\mathrm{Cu}$ nanoparticles with PVA matrix, optical properties such as complex refractive index and dielectric constant have been calculated. The refractive index $n$ of the films was calculated using the following equation [20]:

$n=\left(\frac{1+R}{1-R}\right)+\sqrt{\frac{4 R}{(1-R)^{2}}-k^{2}}$,

In which $k=\frac{\lambda \alpha}{4 \pi}$.

where $\alpha$ is the optical density and $\lambda$ is the wavelength of the light used. Fig. 5 shows the variation of the refractive index of pure PVA and PVA doped nanocomposite films with the photon energy before annealing.

It can be discerned from the graph that the refractive index that was measured at visible wavelengths before annealing became more pronounced for the PVA doped nanocomposite films as compared to pure PVA. The high value of refractive index is an indication of high density of the film, which leads to a reduction of the interatomic spacing [15]. The increase in the refractive index for PVA doped nanocomposite films is significant for materials that could be used for fabrication of optical waveguides. The dependence of the refractive index on the film density can be discussed by the well-known Clausius-Mossotti relation [20].

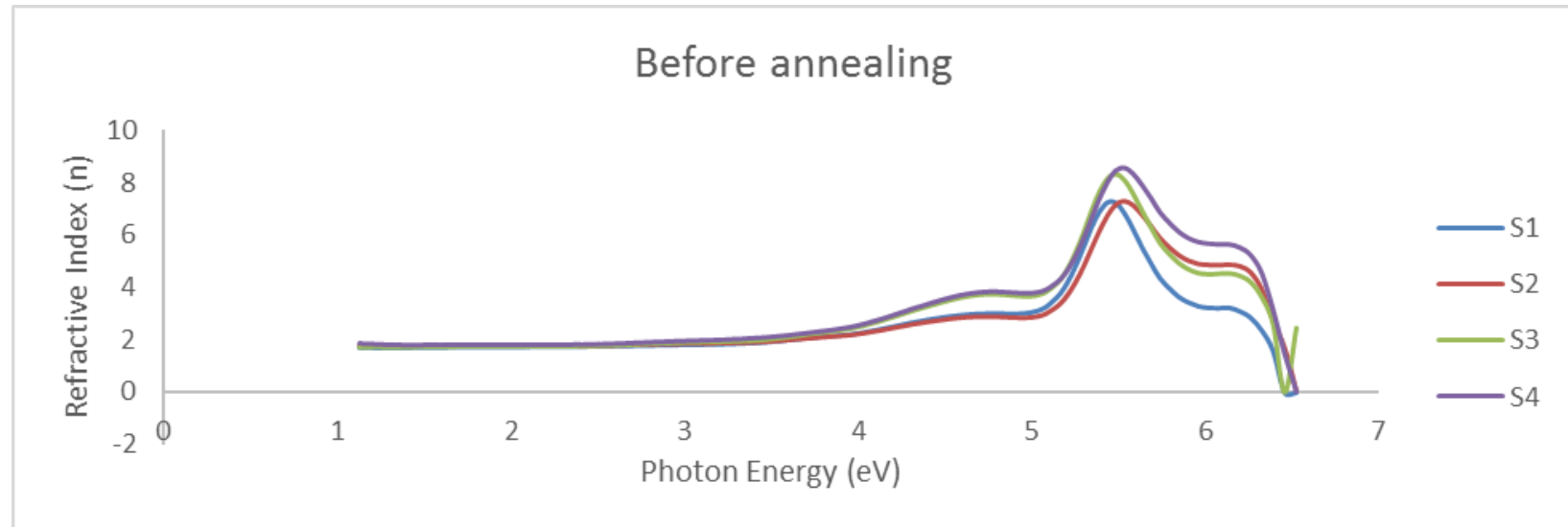

Fig. 5. Variation of refractive index before annealing of pure PVA and PVA doped nanocomposite films.

The change of the extinction coefficient as a function of the photon energy before annealing is shown in figure (6) for pure PVA and PVA doped nanocomposite films. It can be noted that $(\mathrm{k})$ has low value at low 
concentration, but it increases with the increasing of the concentration of nanoparticles $(\mathrm{Cu})$.

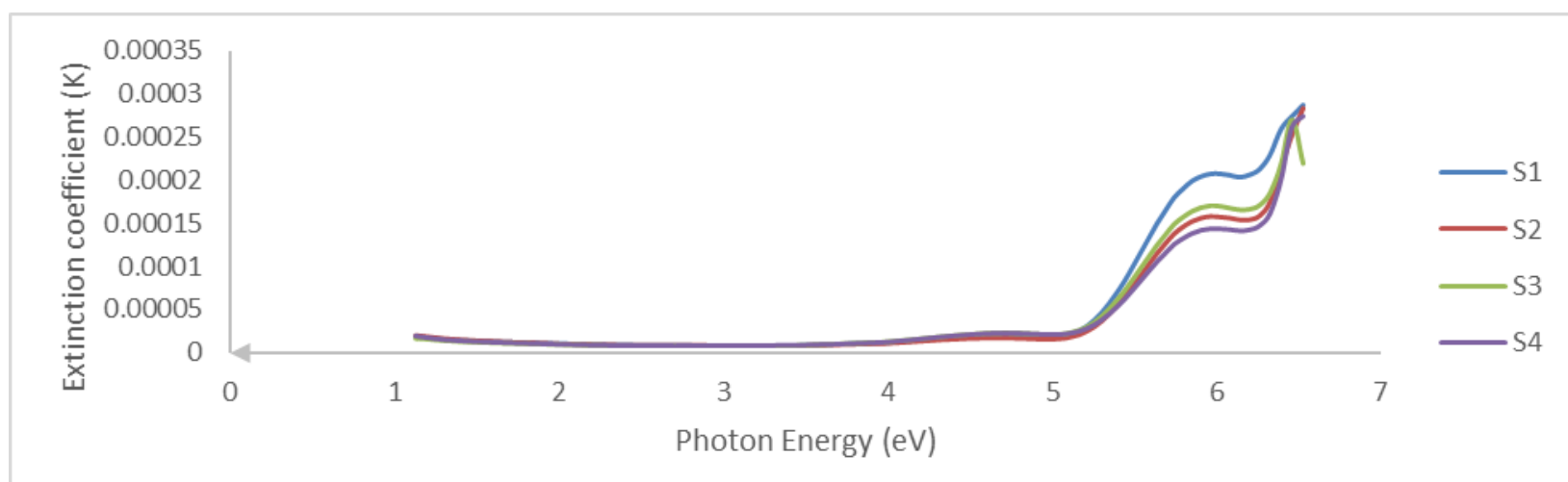

Figure (6): The Extinction coefficient as a function of photon energy for pure PVA and PVA doped nanocomposite films.

The real

$\varepsilon_{r}=\left(n^{2}-k^{2}\right)$

And imaginary

$$
\varepsilon_{i}=2 n k
$$

Parts of optical dielectric constant are also estimated in this work before annealing because these parameters are directly related to the energy density of states within the optical band gap of the samples [21].

The real part of dielectric constant as a function of photon energy before annealing is shown in Fig. (7). The real dielectric constant is increased with the increase of the concentrations of $(\mathrm{Cu})$ nanoparticles.

The imaginary part of dielectric constant as a function of photon energy before annealing is shown in Fig. (8). It is clear from this figure that the imaginary part depends on extinction coefficient according to equation (6) because the refractive index value is very small $[22,23]$. After doping with $\mathrm{Cu}$ the imaginary part of dielectric constant for the samples was increased with the increasing of photon energy and with increasing the doping concentration. 


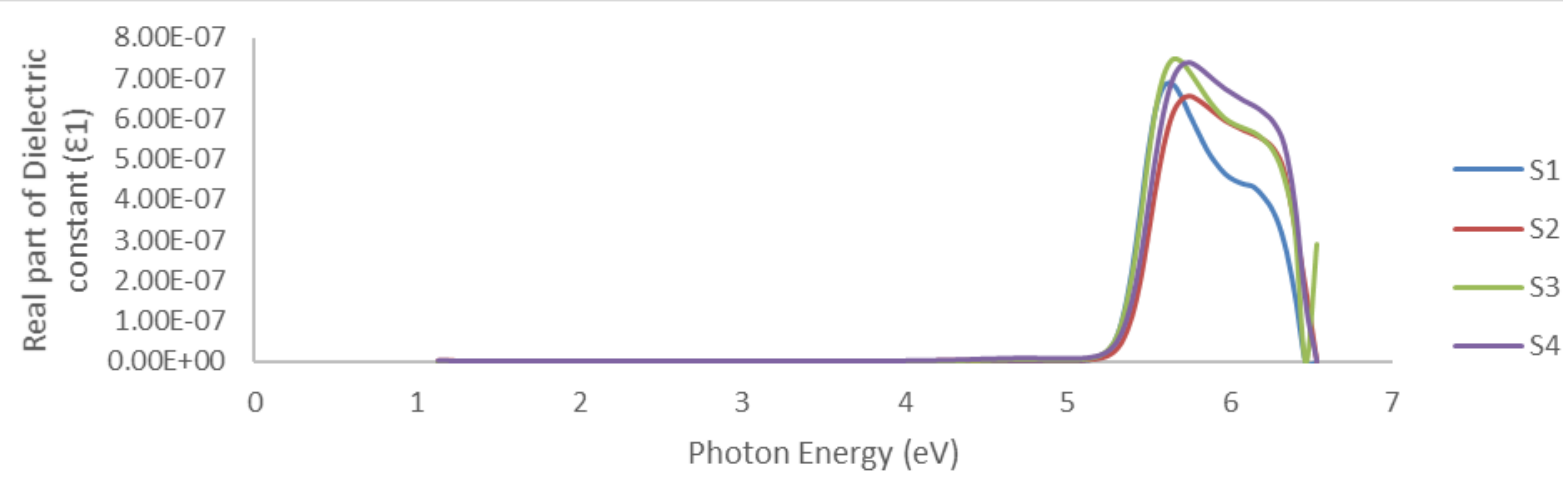

Fig. 7 Optical dielectric constant as a function of photon energy before annealing for pure PVA and PVA doped nanocomposite films.

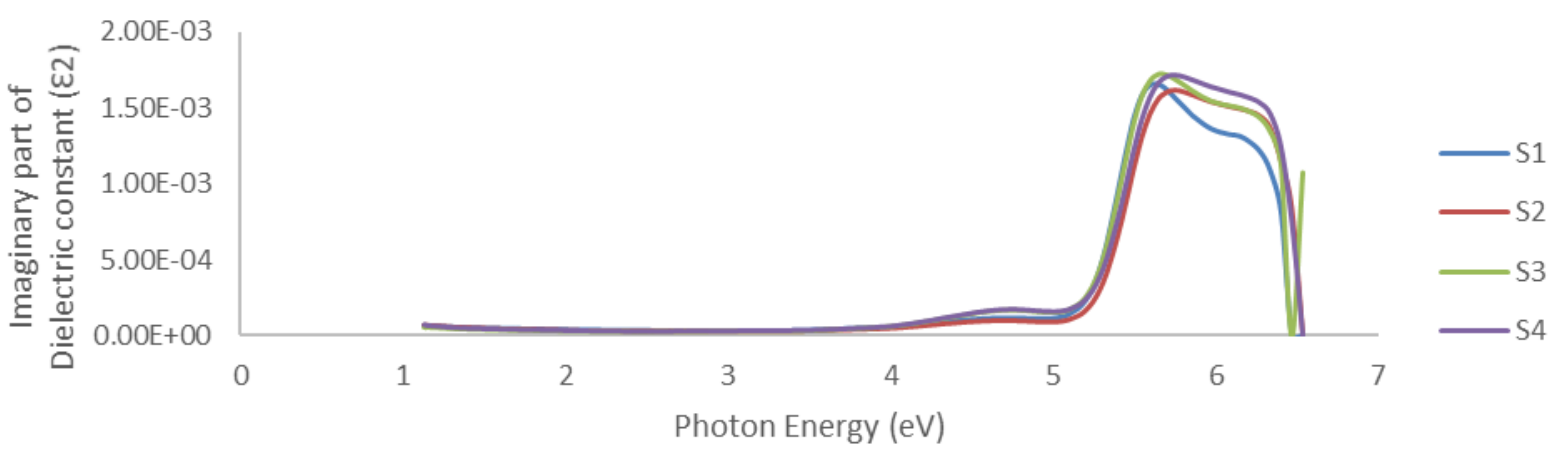

Fig. 8 Optical dielectric loss as a function of photon energy before annealing for pure PVA and PVA doped nanocomposite films.

After annealing the samples for one hour at $75^{\circ} \mathrm{C}$ the optical properties further improved and the calculated optical band gap decreased more for the pure PVA and PVA doped nanocomposite films as shown in figure 9 for S1 and S4. The calculated optical band gaps for the samples before and after annealing are shown in table 1.

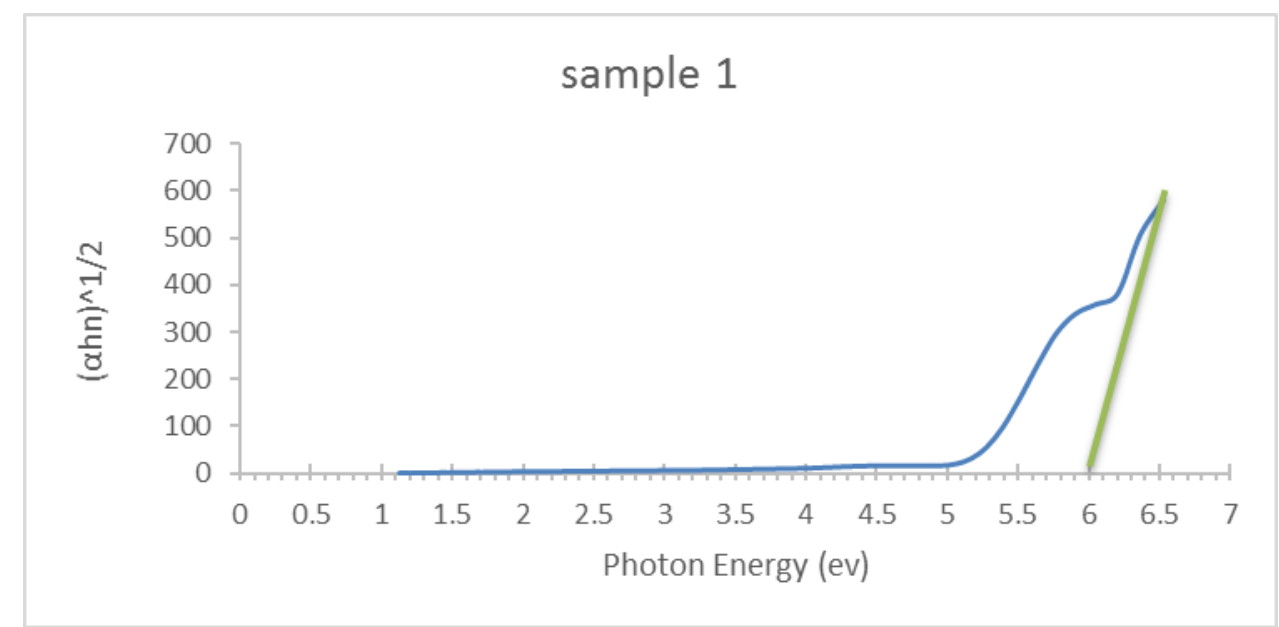




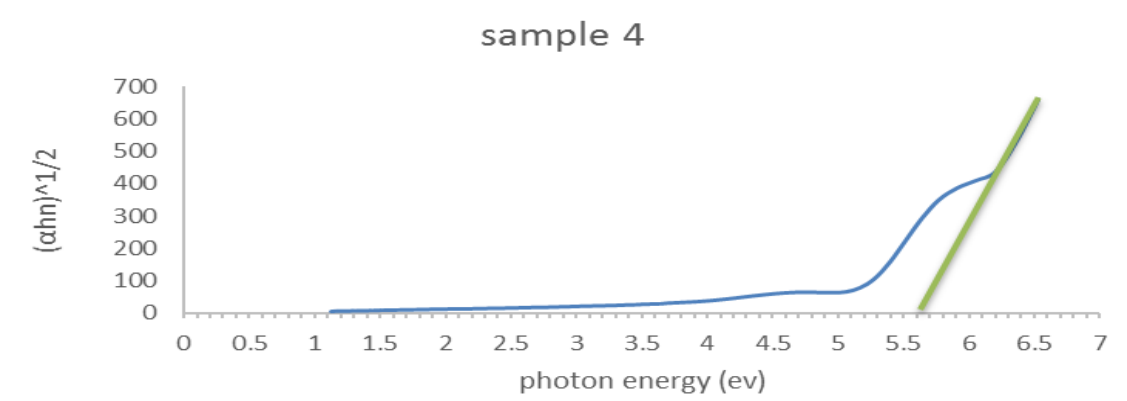

Figure (9): The energy gap after annealing for the allowed indirect transition $(\alpha h v)^{1 / 2}$ as a function of photon energy of S1 and S4 pure PVA and PVA doped nanocomposite film.

\begin{tabular}{|c|c|c|}
\hline Samples & $\begin{array}{c}\text { Calculated Optical energy } \\
\text { gap before annealing (eV) }\end{array}$ & $\begin{array}{c}\text { Calculated Optical energy } \\
\text { gap after annealing (eV) }\end{array}$ \\
\hline S1 & 6.20 & 6.00 \\
\hline S2 & 6.00 & 5.9 \\
\hline S3 & 5.90 & 5.8 \\
\hline S4 & 5.80 & 5.6 \\
\hline
\end{tabular}

Table 1. Calculated Optical energy gap for the pure PVA and PVA doped nanocomposite film before and after annealing.

The variation or comparison of the refractive index of pure PVA and PVA doped nanocomposite film (S1 \& S4) with the photon energy before and after annealing are shown in Figure 10.

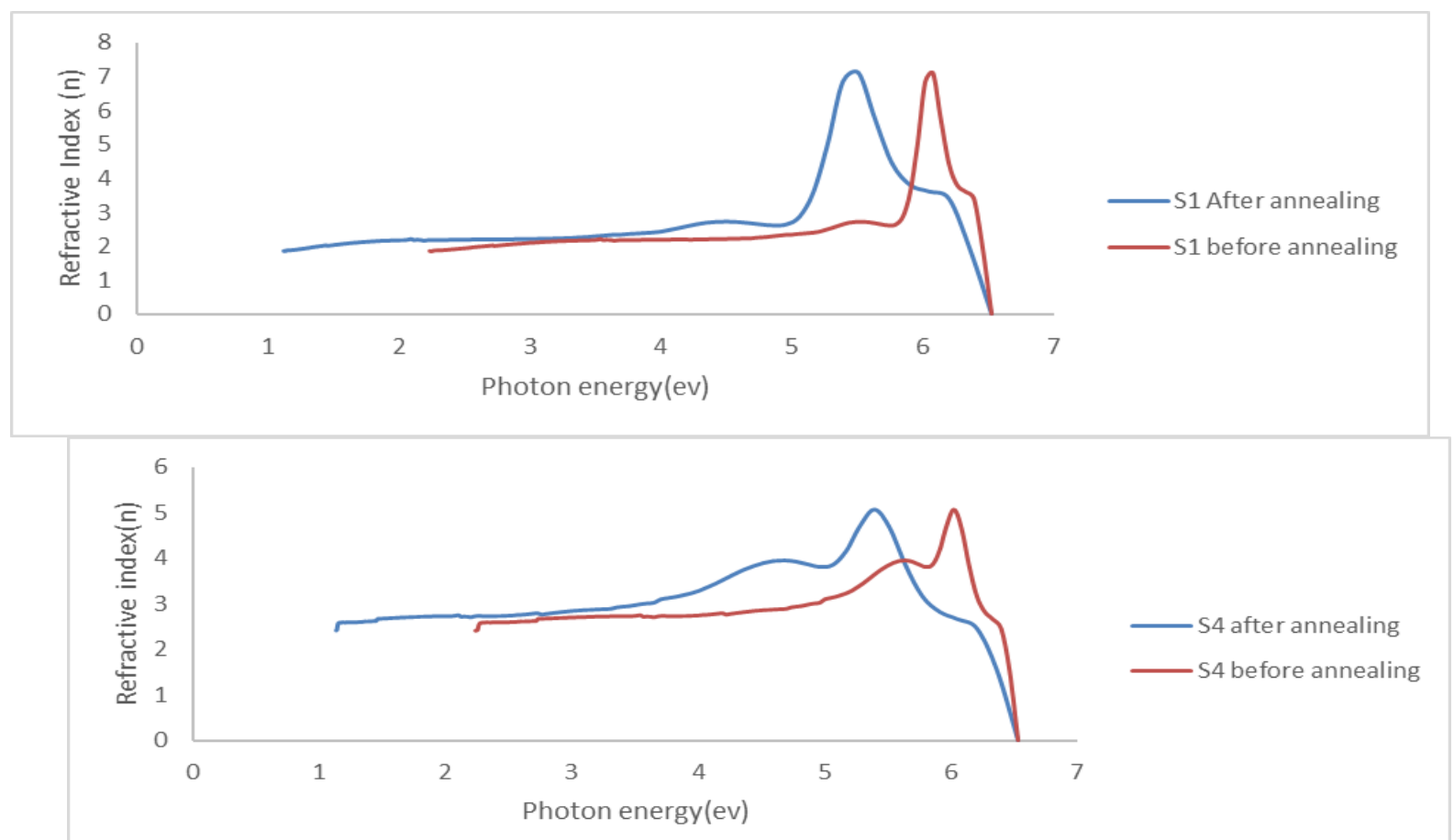

Figure 10 Variation of the Refractive Index for the pure PVA and PVA doped nanocomposite film before and after annealing. 
The obtained XRD spectrums obtained have two main peaks which is common in all composite films. XRD spectrum of the pure PVA and PVA doped nanocomposite films are shown in Figure 11. The diffraction pattern of pure PVA indicates a diffraction bands at $2 \theta=19.565^{\circ}, 40.7^{\circ}$. It is well known that the peaks at $2 \theta<20^{\circ}$ are due to crystalline nature of PVA polymer molecules, which may be as a result of strong intermolecular and intramolecular hydrogen banding between the PVA chains $[24,25]$. The peaks at angles larger than $20^{\circ}$ may be due to impurities.

The intensity of diffracted X-ray photons from films has been increased noticeably after the doping process. It may be due to two reasons. For $2 \theta<20$ increasing the peaks intensity is due to increasing the number of PVA chains in the structure of the films after $\mathrm{Cu}$ doping and increases the intensity of the X-rays reflected from the sample. [24, 25]. For $2 \theta>20$ increasing the intensity of XRD peaks is due to increasing the number of crystallographic planes at certain angles.

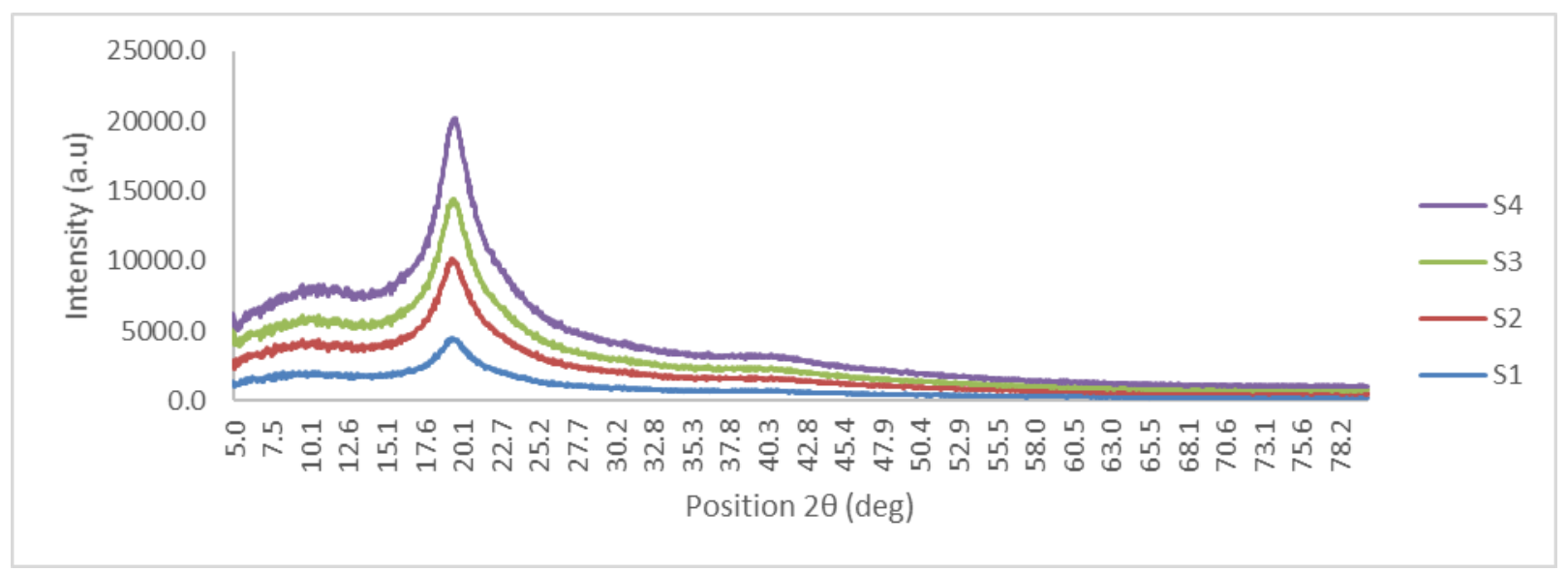

Figure 11: X-ray diffraction patterns of pure PVA, \& PVA doped nanocomposite films. 


\section{Conclusion}

$\mathrm{Cu}$ NPs can be prepared by laser ablation method at different fluencies of laser pulse in pure water. Optical and structural characterization of synthesized pure PV and PVA doped nanocomposite films were carried out using UV-Visible Spectroscopy, X-ray diffraction (XRD). The experimental results shows that the absorbance and absorption coefficient parameters are greatly affected by variation of copper $(\mathrm{Cu})$ NPs concentration. It was observed that optical band gap of pure PVA is significantly reduced upon the addition of $\mathrm{Cu}$ NPs. The increase of refractive index with increasing $\mathrm{Cu}$ concentration is an evidence for the formation of new energy states and thus decreasing the energy band gap of PVA. The increase of optical dielectric constant was observed upon the addition of $\mathrm{Cu}$ nanoparticles. Appearance of characteristic surface Plasmon resonance peak of $\mathrm{Cu}$ nanoparticles at $591 \mathrm{~nm}$ in absorption spectra of Cu-PVA colloidal solution confirms the formation of $\mathrm{Cu}$ nanoparticles. Analysis of UV-Visible absorption and reflection data indicates towards the reduction in optical band gap and increase in refractive index of the resulting nano-composite.

After annealing the samples for one hour at $75^{\circ} \mathrm{C}$ the optical properties improved further.

XRD spectrum of the pure PVA and PVA doped nanocomposite films show that the intensity of diffracted X-ray photons from films has been increased noticeably after the doping process.

\section{References}

[1] S. Mahendia, A. K. Tomar, and S. Kumar, "Electrical conductivity and dielectric spectroscopic studies of PVA-Ag nano composite films," Journal of Alloys and Compounds, vol. 508, no. 2, pp. 406-411, 2010.

[2] P. K. Khanna, R. Gokhale, V. V. V. S. Subbarao, A. K. Vish- wanath, B. K. Das, and C. V. V. Satyanarayana, "PVA stabilized gold nanoparticles by use of 
unexplored albeit conventional reducing agent," Materials Chemistry and Physics, vol . 92, no. 1, pp. 229-233, 2005.

[3] G. Nesher, G. Marom, and D. Avnir, "Metal-polymer composites: synthesis and characterization of polyaniline and other polymer at Silver compositions," Chemistry of Materials, vol. 20, no. 13, pp. 4425-4432, 2008.

[4] O. L. A. Monti, J. T. Fourkas, and D. J. Nesbitt, "Diffraction- limited photo generation and characterization of silver nanoparticles," Journal of Physical Chemistry B, vol. 108, no. 5, pp. 1604-1612, 2004.

[5] K. L. Kelly, E. Coronado, L. L. Zhao, and G. C. Schatz, "The optical properties of metal nanoparticles: the influence of size, shape, and dielectric environment," Journal of Physical Chemistry B, vol. 107, no. 3, pp. 668-677, 2003.

[6] A. A. Scalisi, G. Compagnini, L. D’Urso, and O. Puglisi, "Non- linear optical activity in $\mathrm{Ag}-\mathrm{SiO} 2$ nano composite thin films with different silver concentration," Applied Surface Science, vol. 226, no. 1-3, pp. 237-241, 2004.

[7] H. B. Liao, R. F. Xiao, H. Wang, K. S. Wong, and G. K. L. Wong, "Large thirdorder optical nonlinearity in $\mathrm{Au}: \mathrm{TiO} 2$ composite films measured on a femtosecond time scale," Applied Physics Letters, vol. 72, no. 15, pp. 1817-1819, 1998.

[8] A. K. Sarychev, D. J. Bergman, and Y. Yagil, "Theory of the optical and microwave properties of metal-dielectric films," Physical Review B, vol. 51, no. 8, pp. 5366-5385, 1995.

[9] S. Link and M. A. El-Sayed, "Spectral properties and relaxation dynamics of surface Plasmon electronic oscillations in gold and silver nano dots and nano rods," Journal of Physical Chemistry B, vol. 103, no. 40, pp. 8410-8426, 1999.

[10] A. Nimrodh Ananth and S. Umapathy, "On the optical and thermal properties of in situ/ex situ reduced Ag NP's/PVA composites and its role as a simple SPRbased protein sensor," Applied Nano science, vol. 1, no. 2, pp. 87-96, 2011.

[11] G. Fussell, J. Thomas, J. Scanlon, A. Lowman, and M. Marcolongo, "The effect of protein-free versus protein-containing medium on the mechanical 
properties and uptake of ions of PVA/PVP hydrogels," Journal of Biomaterials Science, vol. 16, no. 4, pp. 489-503, 2005.

[12] J. Brandrup, E.H. Immergut, E.A. Grulke, A. Abe, D.R. Bloch, Polymer Handbook, John Wiley and Sons, 2005.

[13] D.M. Fernandes, J.L. Andrade, M.K. Lima, M.F. Silva, L.H.C. Andrade, S.M. Lima, A.A.W. Hechenleitner, E.A.G. Pineda, Thermal and photochemical effects on the structure, morphology, thermal and optical properties of PVA/Ni0.04Zn0.96O and PVA/ Fe0.03Zn0.97O nanocomposite films. Polym. Degrad. Stab. 98, 1862-1868 (2013)

[14] T. Tsuji, T. Mizuki, S. Ozono, and M. Tsuji, "Laser-induced silver nano crystal formation in polyvinyl pyrrolidone solutions," Journal of Photochemistry and Photobiology A, vol. 206, no. 2-3, pp. 134-139, 2009.

[15 M. Abdelaziz, Cerium (III) doping effects on optical and thermal properties of PVA. films, Physica B., Vol.406 (6-7), pp.1300-1307, 2011.

[16] M. Dahshan," Introduction to Material Science and Engineering”, 2nd Ed., 2002.

[17] P.U.Asogwa "Band gap shift and optical characterization of PVA Capped PbO thin films : Effect of thermal annealing " Chalcogenid Lett.Vol.8,No.3 ,pp163-170, 2011.

[18] Han, X.; Liu, R.; Chen, W.; Xu, Z.; "Properties of nano crystalline zinc oxide thin films prepared by thermal decomposition of electrodeposited zinc peroxide"; Thin Solid Films.006, .120-.124, 2110.

[19] Soltani, N.; Gharibshahi, E.; Saion, E. Band gap of cubic and hexagonal CdS quantum dots-experimental and theoretical studies. Chalcogenide Lett. 2012, 9, $321-328$.

[20] C. Kittle, Introduction to Solid State Physics, vol. 405, John Wiley \& Sons, New York, NY, USA, 1971. 
[21] M. Caglar, M. Zor, S. Ilican, Y. Caglar, Effect of indium incorporation on the optical properties of spray pyrolyzed Cd0.22- Zn0.78S thin films. Czech J. Phys. 56, 277-287 (2006)

[22] Khadum, K.A.: "Study of optical constant for cadmium oxide films doped with silver oxide (CdO: AgO) in infrared region; Diala, Jour.32, 0-00, 2114.

[23] Ahmad, A. H.; Awatif, A. M.; Zeid, Abdul-Majied. N.; "Doping effect on optical constants of polymethylmethacrylate (PMMA)"; Eng. \& Technology. 20(.), 000- 060, 2115.

[24] S. Mahendia, A. K. Tomar, and S. Kumar, "Electrical conductivity and dielectric spectroscopic studies of PVA-Ag nanocomposite films" Journal of Alloys and Compounds, vol. 508, no. 2, pp. 406-411, 2010.,

[25] A. Gautam and S. Ram, "Preparation and thermo mechanical properties of AgPVA nanocomposite films," Materials Chemistry and Physics, vol. 119, no. 1-2, pp. 266-271, 2010. 